\title{
Analytical study for Soret, Hall, and Joule heating effects on natural convection flow saturated porous medium in a vertical channel
}

\author{
K. Kaladhar ${ }^{1} \cdot$ Ch. $\operatorname{RamReddy}^{2} \cdot$ D. Srinivasacharya ${ }^{2} \cdot$ T. Pradeepa $^{2}$
}

Received: 30 September 2015/ Accepted: 23 July 2016/Published online: 12 August 2016

(c) The Author(s) 2016. This article is published with open access at Springerlink.com

\begin{abstract}
This paper analyzes the laminar, incompressible natural convective transport inside vertical channel in an electrically conducting fluid saturated porous medium. In addition, this model incorporates the combined effects of Hall current, Soret and Joule heating. The non-linear governing equations with their corresponding boundary conditions are initially cast into a dimensionless form by using suitable transformations and Adomian decomposition method has been used to solve the system of equations. To explore the influence of various parameters on fluid flow properties, quantitative analysis is exhibited graphically and shown in the tabular form.
\end{abstract}

Keywords Natural convection - Soret effect · Hall effect . Joule heating parameter $\cdot$ ADM

\section{Introduction}

Considerable attention has been paid to the immense investigation of natural convection flow saturated porous medium due to its diverse application in engineering and industrial process such as dispersion of fog, solar energy collecting devices, air conditioning of a room, material processing, cooling of molten metals, petroleum industries, moisture transport in thermal insulation etc. Different simulation procedures have been adopted by several

K. Kaladhar

kaladhar@nitpy.ac.in

1 Department of Mathematics, National Institute of Technology Puducherry, Karaikal 609605, India

2 Department of Mathematics, National Institute of Technology Warangal, Warangal, India researchers to analyze the natural convection flow saturated porous medium in vertical channel with various fluid models. The analysis of combined free and forced convection flow between two asymmetrically and symmetrically heated vertical parallel walls in a porous medium with viscous dissipation effect has been considered by Ingham et al. [1]. Paul et al. [2] presented an analytical solution for the free convection flow between vertical walls partially filled with porous matrix and a clear fluid placing an interface vertically. Umavathi [3] discussed the natural convection flow of immiscible fluids in a vertical channel filled with a porous medium taking into account of DarcyBrinkman-Forchheimer equation model. The free convection flow between vertical walls in porous medium with radiation effect has been studied by Mishra et al. [4].

Soret effect (thermal-diffusion) refers to the differentiation of species. Soret effect is neglected in many cases related to the transfer of heat and mass due to that it is a smaller order of magnitude than the effects described by Fourier's and Fick's laws. The applications and early literature can be found in [5]. Srinivasacharya and Kaladhar [6] analyzed the free convection flow of a chemical reacting couple stress fluid in a vertical channel with the influence of Soret and Dufour effects. With the influence of magnetic field on natural convection flow of a power law fluid in a porous medium with stratification, Dufour and Soret effects has been reported by Srinivasacharya et al. [7] (also see the citations therein).

Several researchers combined the MHD flow problems with Hall effect owing that the Hall current effect cannot neglect when the magnetic field strength is strong. in view of applications, Tani [8] considered the steady motion viscous fluid in a channel with Hall effect. Srinivasacharya and Mekonnen [9-11] presented the Hall and ion-slip effects in non-Newtonian fluids. The unsteady free 
convection flow of an electrically conducting fluid through a porous medium in a vertical channel with effects of thermal radiation, thermal diffusion and Hall current has been reported by Manglesh and Gorla [12]. Garg et al. [13] examined the effect of Hall current viscoelastic fluid in a vertical porous channel filled with porous medium and oscillatory magneto-hydrodynamic convection.

In all erstwhile studies, the effect of Joule heating was ignored and works on Joule heating effects of participating MHD are fewer. Joule heating is the predominant heat mechanism for heat generation in integrated circuits and is an undesired effect. Chen [14] considered the radiative heat transfer and free convection flow past permeable stretching surfaces with Joule heating and viscous dissipation effects. Hossain and Gorla [15] scrutinized the effect of Joule heating on mixed convection boundary layer flow of an electrically conducting fluid past a vertical surface in the presence of a uniform transverse magnetic field fixed relative to the surface. The combined viscous and Joule heating effects on nonlinear convection flow on stagnation point through stretching or shrinking sheet in the presence of homogeneous and heterogeneous reactions has been studied by Nandkeolyar [16].

The present investigation carries out the electrically conducting natural convection in a vertical porous medium with the influence of thermal diffusion, Hall current and Joule heating effects. Inspite of the complex structure of the problem and to provide more accurate analysis to the technical and industrial applications, the final nonlinear system of equations are solved using Adomian decomposition method. Interesting features of the present setup has been given at the end of the study.

\section{Formulation of the problem}

Consider a laminar, incompressible electrically conducting fluid saturated porous medium in a vertical channel. The plates are placed vertically upwards (in the direction of $x$-axis) and $y$-axis is perpendicular to the plates. The plates are extended infinitely in directions of $x$ and $z$ and is presented in Fig. 1. The effects of Joule heating and Hall currents produces due to the applied uniform magnetic field (applied in the direction of $y$-axis). The additional force in the $z$-direction is generated due to the effect of Hall current, which gives rise to a cross flow therefore the flow becomes three dimensional. In this present study, the magnetic Reynolds number is very small with that the induced magnetic field has been neglected in comparison with the applied magnetic field. Buoyancy forces causes the natural convection flow. With the above presumptions, the governing equations
Fig. 1 Physical model and coordinate system

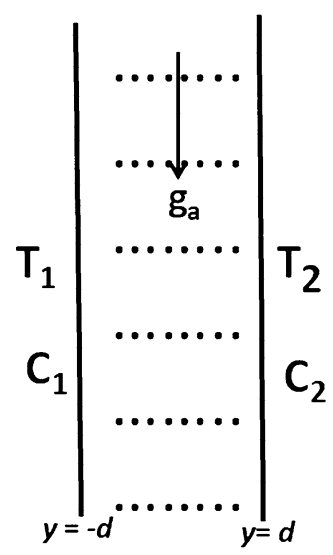

for an electrically conducting fluid saturated porous medium with thermal-diffusion are

$\frac{\partial v}{\partial y}=0 \Rightarrow v=v_{0}=$ constant

$\begin{aligned} \rho v \frac{\partial u}{\partial y}= & \mu \frac{\partial^{2} u}{\partial y^{2}}+\rho g_{\mathrm{a}} \beta_{\mathrm{T}}\left(\left(T-T_{1}\right)+\frac{\beta_{\mathrm{C}}}{\beta_{\mathrm{T}}}\left(C-C_{1}\right)\right) \\ & -\frac{\sigma B_{0}^{2}}{1+\beta_{\mathrm{h}}^{2}}\left(u+\beta_{\mathrm{h}} w\right)-\frac{\varepsilon \mu}{K_{\mathrm{f}}} u\end{aligned}$

$\rho v \frac{\partial w}{\partial y}=\mu \frac{\partial^{2} w}{\partial y^{2}}+\frac{\sigma B_{0}^{2}}{1+\beta_{\mathrm{h}}^{2}}\left(\beta_{\mathrm{h}} u-w\right)-\frac{\varepsilon \mu}{K_{\mathrm{f}}} w$

$\rho C_{\mathrm{p}} v \frac{\partial T}{\partial y}=K_{\mathrm{f}} \frac{\partial^{2} T}{\partial y^{2}}+\mu\left[\left(\frac{\partial u}{\partial y}\right)^{2}+\left(\frac{\partial w}{\partial y}\right)^{2}\right]$ $+\frac{\sigma B_{0}^{2}}{1+\beta_{\mathrm{h}}^{2}}\left(u^{2}+w^{2}\right)$

$v \frac{\partial C}{\partial y}=D \frac{\partial^{2} C}{\partial y^{2}}+\frac{D K_{\mathrm{f}}}{T_{\mathrm{m}}} \frac{\partial^{2} T}{\partial y^{2}}$

with

$u=w=0 \quad$ at $\mathrm{y}= \pm \mathrm{d}$

$T=T_{1}, \quad C=C_{1} \quad$ at $\mathrm{y}=-\mathrm{d}$

$T=T_{2}, \quad C=C_{2} \quad$ at $\mathrm{y}=\mathrm{d}$

where the velocity components in $x-, y-$ and $z$ - directions are taken as $u, v, w$ respectively, $g_{\mathrm{a}}$ is the acceleration due to gravity, $\rho$ is the density, $C_{\mathrm{p}}$ is the specific heat, $\mu$ is the coefficient of viscosity, $\varepsilon$ is the porosity, $\beta_{\mathrm{h}}$ is the Hall parameter, $\beta_{\mathrm{T}}$ is the coefficient of thermal expansion, $\beta_{\mathrm{C}}$ is the coefficient of solutal expansion, $\sigma$ is the electric conductivity of the fluid, $B_{0}$ is the uniform magnetic field, $D$ is the mass diffusivity, $K_{\mathrm{f}}$ is the coefficient of thermal conductivity and $T_{\mathrm{m}}$ is the mean fluid temperature. The last terms of (4), (5) represents the Joule heating and thermal diffusions respectively.

Introducing the following dimensionless variables 
$\eta=\frac{y}{d}, \quad u=\frac{\nu \mathrm{Gr}}{d^{2}} f, \quad w=\frac{\nu \mathrm{Gr}}{d^{2}} g$

$\theta=\frac{T-T_{1}}{T_{2}-T_{1}}, \quad \phi=\frac{C-C_{1}}{C_{2}-C_{1}}$

in Eqs. (2)-(5), we obtain the governing dimensionless equations as

$$
\begin{aligned}
& f^{\prime \prime}-\operatorname{Re} f^{\prime}+\theta+N \phi-\frac{\mathrm{Ha}^{2}}{1+\beta_{\mathrm{h}}^{2}}\left(f+\beta_{\mathrm{h}} g\right)-\frac{\epsilon}{\mathrm{Da}} f=0 \\
& g^{\prime \prime}-\operatorname{Re}^{\prime}+\frac{\mathrm{Ha}^{2}}{1+\beta_{\mathrm{h}}^{2}}\left(\beta_{\mathrm{h}} f-g\right)-\frac{\epsilon}{\mathrm{Da}} g=0 \\
& \theta^{\prime \prime}-\operatorname{RePr} \theta^{\prime}+\mathrm{BrGr}^{2}\left[\left(f^{\prime}\right)^{2}+\left(g^{\prime}\right)^{2}\right] \\
& \quad+\frac{J}{1+\beta_{\mathrm{h}}^{2}} \mathrm{Gr}^{2}\left(f^{2}+g^{2}\right)=0 \\
& \phi^{\prime \prime}-\operatorname{ReSc} \phi^{\prime}+\operatorname{ScSr} \theta^{\prime \prime}=0
\end{aligned}
$$

with

$f=g=\theta=\phi=0 \quad$ at $\quad \eta=-1$

$f=g=0, \theta=\phi=1 \quad$ at $\quad \eta=1$

where the primes denote differentiation with respect to $\eta$, $\mathrm{Ha}=B_{0} d \sqrt{\frac{\sigma}{\mu}}$ is the Hartmann parameter, $N=\frac{\beta_{\mathrm{C}}\left(C_{2}-C_{1}\right)}{\beta_{\mathrm{T}}\left(T_{2}-T_{1}\right)}$ is the buoyancy parameter, $\operatorname{Re}=\frac{\rho v_{0} d}{\mu}$ is the Reynolds number, $\mathrm{Gr}=\frac{g_{\mathrm{a}} \beta_{\mathrm{T}}\left(T_{2}-T_{1}\right) d^{3}}{v^{2}}$ is the Grashof number, $\operatorname{Pr}=\frac{\mu C_{\mathrm{p}}}{K_{\mathrm{f}}}$ is the Prandtl number, $\mathrm{Br}=\frac{\mu v^{2}}{K_{\mathrm{f}} d^{2}\left(T_{2}-T_{1}\right)}$ is the Brinkman parameter, $J=\mathrm{Ha}^{2} \mathrm{Br}$ is the Joule heating parameter and $S_{r}=$ $\frac{D K_{\mathrm{T}}\left(T_{2}-T_{1}\right)}{v T_{\mathrm{m}}\left(C_{2}-C_{1}\right)}$ is the thermo-diffusion parameter.

The shearing stress, heat and mass fluxes at the wall surfaces can be obtained from

$$
\begin{array}{r}
\left.\tau_{w}=\left.\mu \frac{\partial u}{\partial y}\right|_{y= \pm d} ; q_{w}=-K_{\mathrm{f}} \frac{\partial T}{\partial y}\right]_{y= \pm d} ; \\
\left.q_{\mathrm{m}}=-D \frac{\partial C}{\partial y}\right]_{y= \pm d}
\end{array}
$$

The non-dimensional shear stress $C_{\mathrm{f}}=\frac{\tau_{w}}{\rho u_{0}^{2}}$, the Nusselt number $\mathrm{Nu}=\frac{q_{w} d}{K_{\mathrm{f}}\left(T_{2}-T_{1}\right)}$ and the Sherwood number $\mathrm{Sh}=\frac{q_{\mathrm{m}} d}{D\left(C_{2}-C_{1}\right)}$ are given by

$$
\begin{aligned}
\operatorname{Re}_{\mathrm{f} 1} & =2 f^{\prime}(-1) ; \operatorname{Re} C_{\mathrm{f} 2}=2 f^{\prime}(1) \\
\mathrm{Nu}_{1,2} & =-\left.\theta^{\prime}(\eta)\right|_{\eta=-1,1} ; \quad \operatorname{Sh}_{1,2}=-\left.\phi^{\prime}(\eta)\right|_{\eta=-1,1}
\end{aligned}
$$

\section{The ADM solution}

Consider the equation $F u(t)=g(t)$, where $F$ represents a general nonlinear ordinary or partial differential operator including both linear and nonlinear terms. The main idea of the ADM is that decomposing the linear terms into $L+R$ and rewriting the equations into the following form

$(L+R) u+N u=g$

where $L$ is easily invertible(usually the highest order derivative), $R$ is the remained of the linear operator and $\mathrm{Nu}$ indicates the nonlinear terms.

By solving this equation for $L u$, since $L$ is invertible, we can write

$L^{-1} L u=L^{-1} g+L^{-1} R u+L^{-1} N u$

If $L$ is a second-order operator, $L^{-1}$ is a twofold indefinite integral. By solving Eq. (14), we have

$u=A+B t+L^{-1} g+L^{-1} R u+L^{-1} N u$

where $A$ and $B$ are integration constants and can be obtained from the initial or boundary conditions. Adomian decomposition method assumes the solution $u$ that can be expanded into infinite series as

$u=\sum_{n=0}^{\infty} u_{n}$

Also, the nonlinear term $N u$ will be written as

$N u=\sum_{n=0}^{\infty} A_{n}$

where $A_{n}^{\prime} s$ are the special Adomian polynomials. By specified $A_{n}^{\prime} s$, next component of $u$ can be determined.

$u_{n+1}=L^{-1} \sum_{n=0}^{\infty} A_{n}$

After getting the considerable accuracy, the solution is presented in Eq. (15). In Eq. (18), the Adomian polynomials can be generated by several means. Here we used the following recursive formulation

$A_{n}=\frac{1}{n !}\left[\frac{\mathrm{d}^{\mathrm{n}}}{\mathrm{d} \lambda^{\mathrm{n}}}\left[N\left(\sum_{i=0}^{n} \lambda^{i} u_{i}\right)\right]\right]_{\lambda=0}, \quad n=0,1,2, \ldots$

The calculated solution is more realistic than those achieved by simplifying the model of the physical problem since it does not resort to assumption of weak nonlinearity or linearization. 
According to Eq. (14), the governing Eqs. (8)-(11) must be written as following

$$
\begin{aligned}
L_{1} f= & \operatorname{Re} f^{\prime}-\theta-N \phi+\frac{H_{\mathrm{a}}^{2}}{1+\beta_{\mathrm{h}}^{2}}\left(f+\beta_{\mathrm{h}} g\right)+\frac{\epsilon}{\mathrm{Da}} f \\
L_{2} g= & \operatorname{Re} g^{\prime}-\frac{H_{\mathrm{a}}^{2}}{1+\beta_{\mathrm{h}}^{2}}\left(\beta_{\mathrm{h}} f-g\right)+\frac{\epsilon}{\mathrm{Da}} g \\
L_{3} \theta= & \operatorname{Re} \operatorname{Pr} \theta^{\prime}-\operatorname{BrGr}^{2}\left[\left(f^{\prime}\right)^{2}+\left(g^{\prime}\right)^{2}\right] \\
& -\frac{J}{1+\beta_{h}^{2}} \operatorname{Gr}^{2}\left(f^{2}+g^{2}\right)
\end{aligned}
$$

$$
L_{4} \phi=\operatorname{Re} \operatorname{Sc} \phi^{\prime}-\operatorname{ScSr} \theta^{\prime \prime}
$$

where the differential operator $L_{1}, L_{2}, L_{3}$ and $L_{4}$ are given by $L_{1}=L_{2}=L_{3}=L_{4}=\frac{\mathrm{d}^{2}}{\mathrm{~d} \eta^{2}}$. Assume the inverse of the operator $L_{1}^{-1}, L_{2}^{-1}, L_{3}^{-1}$ and $L_{4}^{-1}$ exists and it can be integrated from 0 to $\eta$. i.e. $L_{1}^{-1}=L_{2}^{-1}=L_{3}^{-1}=L_{4}^{-1}=$ $\int_{0}^{\eta} \int_{0}^{\eta}(.) \mathrm{d} \eta \mathrm{d} \eta$.

After operating $L_{1}^{-1}, L_{2}^{-1}, L_{3}^{-1}, L_{4}^{-1}$ on Eqs. (20)-(23) and exerting boundary condition on it, we have

$$
\begin{aligned}
& f(\eta)=f(0)+f^{\prime}(0) \eta+L_{1}^{-1}\left(N_{1} f\right) \\
& g(\eta)=g(0)+g^{\prime}(0) \eta+L_{2}^{-1}\left(N_{2} g\right) \\
& \theta(\eta)=\theta(0)+\theta^{\prime}(0) \eta+L_{3}^{-1}\left(N_{3} \theta\right) \\
& \phi(\eta)=\phi(0)+\phi^{\prime}(0) \eta+L_{4}^{-1}\left(N_{4} \phi\right)
\end{aligned}
$$

where

$$
\begin{aligned}
& N_{1} f=\operatorname{Re} f^{\prime}-\theta-N \phi+\frac{H_{\mathrm{a}}^{2}}{1+\beta_{\mathrm{h}}^{2}}\left(f+\beta_{\mathrm{h}} g\right)+\frac{\epsilon}{\mathrm{Da}} f ; \\
& N_{2} g=\operatorname{Re} g^{\prime}-\frac{H_{\mathrm{a}}^{2}}{1+\beta_{\mathrm{h}}^{2}}\left(\beta_{\mathrm{h}} f-g\right)+\frac{\epsilon}{\mathrm{Da}} g \\
& N_{3} \theta=\operatorname{Re} \operatorname{Pr} \theta^{\prime}-\mathrm{BrGr}^{2}\left[\left(f^{\prime}\right)^{2}+\left(g^{\prime}\right)^{2}\right]
\end{aligned}
$$$$
-\frac{J}{1+\beta_{h}^{2}} \mathrm{Gr}^{2}\left(f^{2}+g^{2}\right)
$$

$N_{4} \phi=\operatorname{Re} \operatorname{Sc} \phi^{\prime}-\operatorname{Sc} \operatorname{Sr} \theta^{\prime \prime}$

The ADM introduced the following expression

$$
\begin{aligned}
& f(\eta)=\sum_{m=0}^{\infty} f_{m}(\eta)=f_{0}(\eta)+L_{1}^{-1}\left(N_{1} f\right) \\
& g(\eta)=\sum_{m=0}^{\infty} g_{m}(\eta)=g_{0}(\eta)+L_{2}^{-1}\left(N_{2} g\right) \\
& \theta(\eta)=\sum_{m=0}^{\infty} \theta_{m}(\eta)=\theta_{0}(\eta)+L_{3}^{-1}\left(N_{3} \theta\right) \\
& \phi(\eta)=\sum_{m=0}^{\infty} \phi_{m}(\eta)=\phi_{0}(\eta)+L_{4}^{-1}\left(N_{4} \phi\right)
\end{aligned}
$$

To determine the components of $f_{m}(\eta), g_{m}(\eta), \theta_{m}(\eta)$ and $\phi_{m}(\eta)$, the initial values of $f_{0}(\eta), g_{0}(\eta), \theta_{0}(\eta)$ and $\phi_{0}(\eta)$ are defined by applying the boundary conditions

$$
\begin{array}{ll}
f_{0}(\eta)=a_{1}+a_{2} \eta, & g_{0}(\eta)=a_{3}+a_{4} \eta, \\
\theta_{0}(\eta)=a_{5}+a_{6} \eta, & \phi_{0}(\eta)=a_{7}+a_{8} \eta
\end{array}
$$

and

$$
\begin{aligned}
f_{1}(\eta)= & {\left[\operatorname{Re} a_{2}-a_{5}-N a_{7}+\frac{H_{\mathrm{a}}^{2}}{1+\beta_{\mathrm{h}}^{2}}\left(a_{1}+\beta_{\mathrm{h}} a_{3}\right)+\epsilon \frac{a_{1}}{\mathrm{Da}}\right] \frac{\eta^{2}}{2} } \\
& +\left[-a_{6}-N a_{8}+\frac{H_{\mathrm{a}}^{2}}{1+\beta_{\mathrm{h}}^{2}}\left(a_{2}+\beta_{\mathrm{h}} a_{4}\right)+\epsilon \frac{a_{2}}{\mathrm{Da}}\right] \frac{\eta^{3}}{6}
\end{aligned}
$$

$$
\begin{aligned}
g_{1}(\eta)= & {\left[\operatorname{Re} a_{4}-\frac{H_{\mathrm{a}}^{2}}{1+\beta_{\mathrm{h}}^{2}}\left(\beta_{\mathrm{h}} a_{1}-a_{3}\right)+\epsilon \frac{a_{3}}{\mathrm{Da}}\right] \frac{\eta^{2}}{2} } \\
& +\left[\epsilon \frac{a_{4}}{\mathrm{Da}}-\frac{H_{\mathrm{a}}^{2}}{1+\beta_{\mathrm{h}}^{2}}\left(\beta_{\mathrm{h}} a_{2}+a_{4}\right)\right] \frac{\eta^{3}}{6} \\
\theta_{1}(\eta)= & -\frac{J}{1+\beta_{\mathrm{h}}^{2}} \mathrm{Gr}^{2}\left(2 a_{1} a_{2}+2 a_{3} a_{4}\right) \frac{\eta^{3}}{6} \\
& \times\left[\operatorname{Re} \operatorname{Pr}_{6}-\operatorname{BrGr}^{2}\left(a_{2}^{2}+a_{4}^{2}\right)-\frac{J}{1+\beta_{\mathrm{h}}^{2}} \operatorname{Gr}^{2}\left(a_{2}^{2}+a_{4}^{2}\right)\right] \frac{\eta^{2}}{2} \\
& -\frac{J}{1+\beta_{h}^{2}} \operatorname{Gr}^{2}\left(a_{2}^{2}+a_{4}^{2}\right) \frac{\eta^{4}}{12}
\end{aligned}
$$

$$
\phi_{1}(\eta)=\left(\operatorname{Re} \operatorname{Sc} a_{8}\right) \frac{\eta^{2}}{2}
$$

and $f_{m}(\eta), g_{m}(\eta), \theta_{m}(\eta)$ and $\phi_{m}(\eta)$ for $m \geq 2$ be determined in similar way.

Then using the above in the following series expansions

$$
\begin{gathered}
f(\eta)=\sum_{m=0}^{\infty} f_{m}(\eta), g(\eta)=\sum_{m=0}^{\infty} g_{m}(\eta) \\
\theta(\eta)=\sum_{m=0}^{\infty} \theta_{m}(\eta), \phi(\eta)=\sum_{m=0}^{\infty} \phi_{m}(\eta)
\end{gathered}
$$

lead to following equations

$$
\begin{aligned}
f(\eta)= & a_{1}+a_{2} \eta \\
& +\left[\operatorname{Re} a_{2}-a_{5}-N a_{7}+\frac{H_{\mathrm{a}}^{2}}{1+\beta_{\mathrm{h}}^{2}}\left(a_{1}+\beta_{\mathrm{h}} a_{3}\right)+\epsilon \frac{a_{1}}{\mathrm{Da}}\right] \frac{\eta^{2}}{2} \\
& +\left[-a_{6}-N a_{8}+\frac{H_{\mathrm{a}}^{2}}{1+\beta_{\mathrm{h}}^{2}}\left(a_{2}+\beta_{\mathrm{h}} a_{4}\right)+\epsilon \frac{a_{2}}{\mathrm{Da}}\right] \frac{\eta^{3}}{6}+\cdots
\end{aligned}
$$




$$
\begin{aligned}
& g(\eta)=a_{3}+a_{4} \eta \\
& +\left[\operatorname{Re} a_{4}-\frac{H_{\mathrm{a}}^{2}}{1+\beta_{\mathrm{h}}^{2}}\left(\beta_{\mathrm{h}} a_{1}-a_{3}\right)+\epsilon \frac{a_{3}}{\mathrm{Da}}\right] \frac{\eta^{2}}{2} \\
& +\left[\epsilon \frac{a_{4}}{\mathrm{Da}}-\frac{H_{\mathrm{a}}^{2}}{1+\beta_{\mathrm{h}}^{2}}\left(\beta_{\mathrm{h}} a_{2}+a_{4}\right)\right] \frac{\eta^{3}}{6}+\cdots \\
& \theta(\eta)=a_{5}+a_{6} \eta-\frac{J}{1+\beta_{h}^{2}} \operatorname{Gr}^{2}\left(2 a_{1} a_{2}+2 a_{3} a_{4}\right) \frac{\eta^{3}}{6} \\
& +\left[\operatorname{Re} \operatorname{Pr} a_{6}-\operatorname{BrGr}^{2}\left(a_{2}^{2}+a_{4}^{2}\right)\right] \frac{\eta^{2}}{2} \\
& -\left[\frac{J}{1+\beta_{\mathrm{h}}^{2}} \mathrm{Gr}^{2}\left(a_{2}^{2}+a_{4}^{2}\right)\right] \frac{\eta^{2}}{2} \\
& -\frac{J}{1+\beta_{\mathrm{h}}^{2}} \mathrm{Gr}^{2}\left(a_{2}^{2}+a_{4}^{2}\right) \frac{\eta^{4}}{12}+\cdots \\
& \phi(\eta)=a_{7}+a_{8} \eta-\left[\operatorname{ReSc} a_{8}\right] \frac{\eta^{2}}{2} \\
& +\left[R^{2} \mathrm{Sc}^{2} a_{8}+\mathrm{ScSr} \frac{J}{1+\beta_{\mathrm{h}}^{2}} \operatorname{Gr}^{2}\left(2 a_{1} a_{2}+2 a_{3} a_{4}\right)\right] \frac{\eta^{3}}{6} \\
& -\operatorname{Sc} \operatorname{Sr}\left(\operatorname{Re} \operatorname{Pr}, a_{6}-\operatorname{BrGr}^{2}\left(a_{2}^{2}+a_{4}^{2}\right)\right) \frac{\eta^{2}}{2} \\
& +\operatorname{ScSr}\left(\frac{J}{1+\beta_{\mathrm{h}}^{2}} \operatorname{Gr}^{2}\left(a_{1}^{2}+a_{3}^{2}\right)\right) \frac{\eta^{2}}{2} \\
& +\operatorname{ScSr} \frac{J}{1+\beta_{\mathrm{h}}^{2}} \operatorname{Gr}^{2}\left(a_{2}^{2}+a_{4}^{2}\right) \frac{\eta^{4}}{12} \ldots
\end{aligned}
$$

$\mathrm{BY}$ increasing the number of terms, the ADM gives the more accurate solution. For the complete solution of

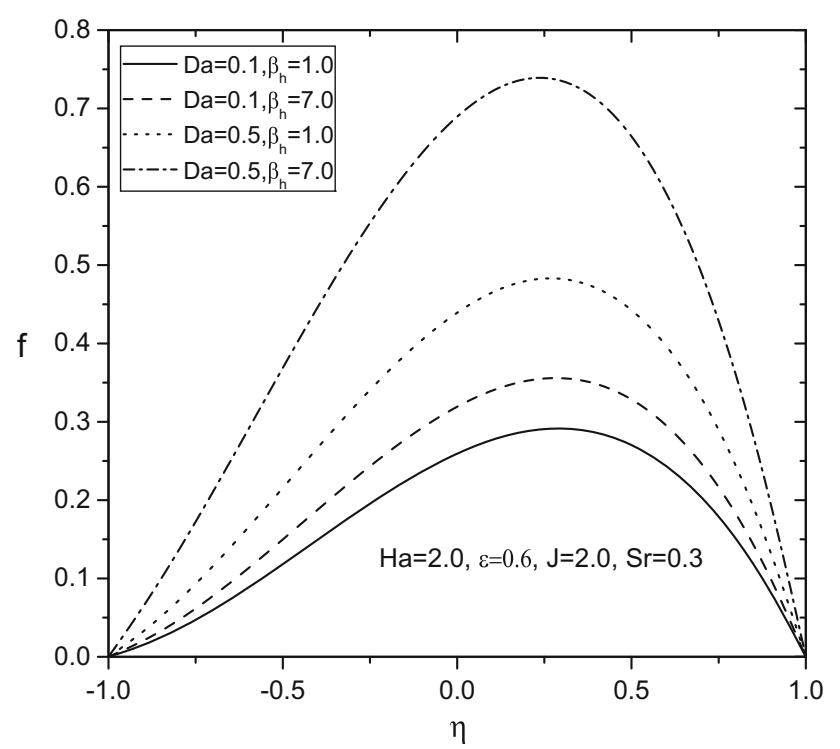

Fig. 2 Effect of Da and $\beta_{\mathrm{h}}$ on velocity profile equations above $a_{1}, a_{2}, a_{3}, a_{4}, a_{5}, a_{6}, a_{7}$ and $a_{8}$ should be determined, with boundary conditions.

\section{Results and discussion}

The velocities $(f(\eta), g(\eta))$, temperature $(\theta(\eta))$ and concentration $(\phi(\eta))$ profiles of are calculated and shown in Figs. 2, 3, 4, 5, 6, 7, 8, 9, 10, 11, 12 and 13 with different values of $\mathrm{Ha}, \beta_{\mathrm{h}}, \mathrm{Sr}, J$ and $\epsilon$, Da. Computations were carried out by fixing the parameters

$\operatorname{Re}=2.0, N=5, \operatorname{Pr}=0.71, \mathrm{Gr}=1.0$ and $\mathrm{Sc}=0.22$

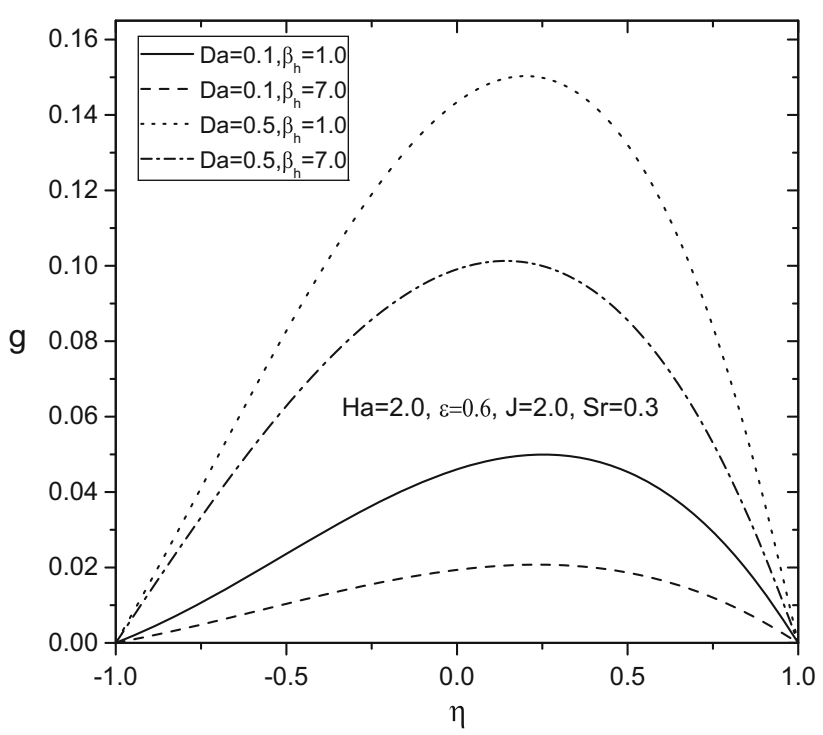

Fig. 3 Effect of Da and $\beta_{\mathrm{h}}$ on angular velocity profile

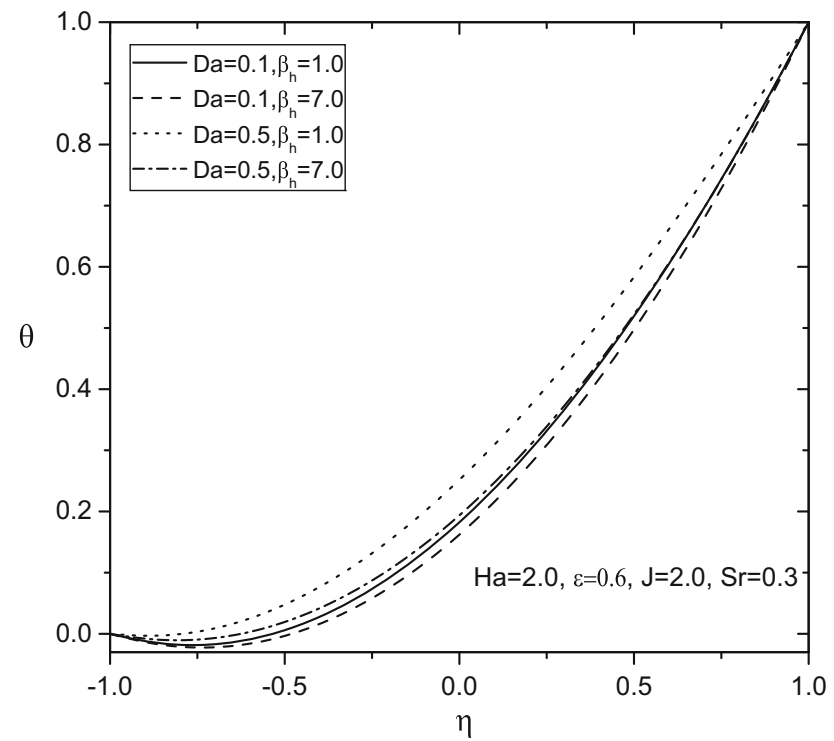

Fig. 4 Effect of Da and $\beta_{\mathrm{h}}$ on temperature profile 


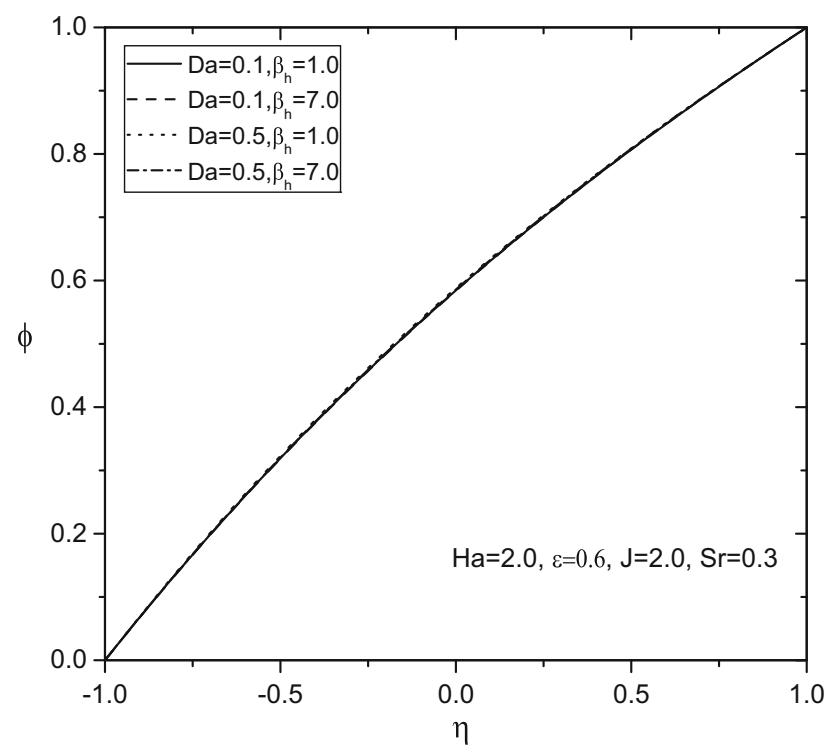

Fig. 5 Effect of Da and $\beta_{\mathrm{h}}$ on concentration profile

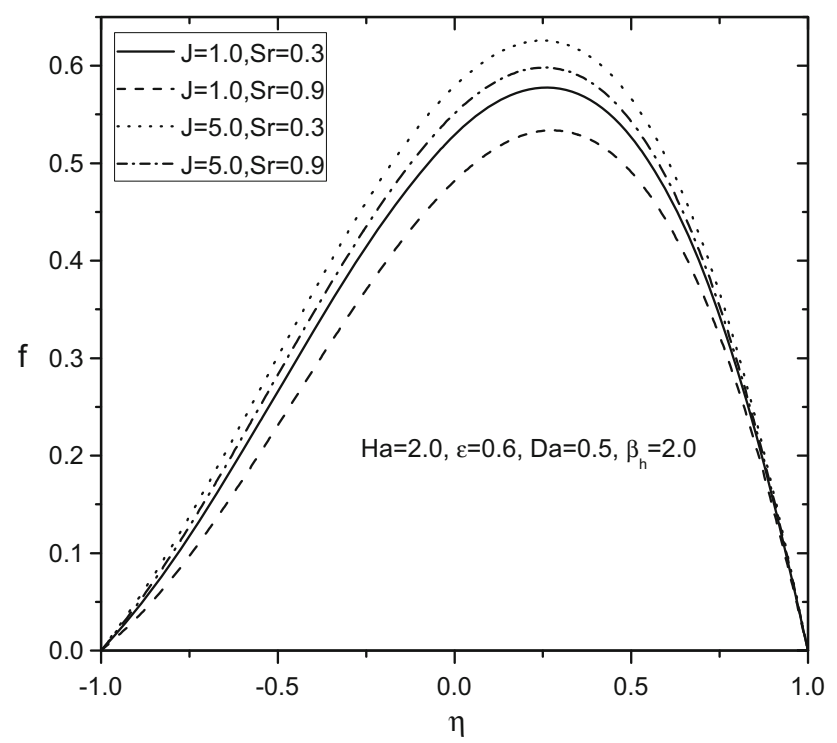

Fig. 6 Effect of $J$ and $\mathrm{Sr}$ on velocity profile

to analyze the effects of the emerging parameters $\mathrm{Ha}, \beta_{\mathrm{h}}$, Sr, Da, $J$ and $\epsilon$.

The effect of the hall parameter $\beta_{\mathrm{h}}$ on velocities, temperature and concentration profiles are shown in Figs. 2, 3, 4 and 5. It can be observed from these figures that $f(\eta)$ increases as $\beta_{\mathrm{h}}$ increases. It is noticed from Fig. 3 that the velocity in $z$-direction decreases with an increase in $\beta_{\mathrm{h}}$. This is due to the Hall parameter's inclusion and which reduces the resistive force imposed by the magnetic field due to its effect in reducing the effective conductivity. It can be seen from Fig. 4 that the temperature of the fluid decreases with an increase in the Hall parameter. Finally

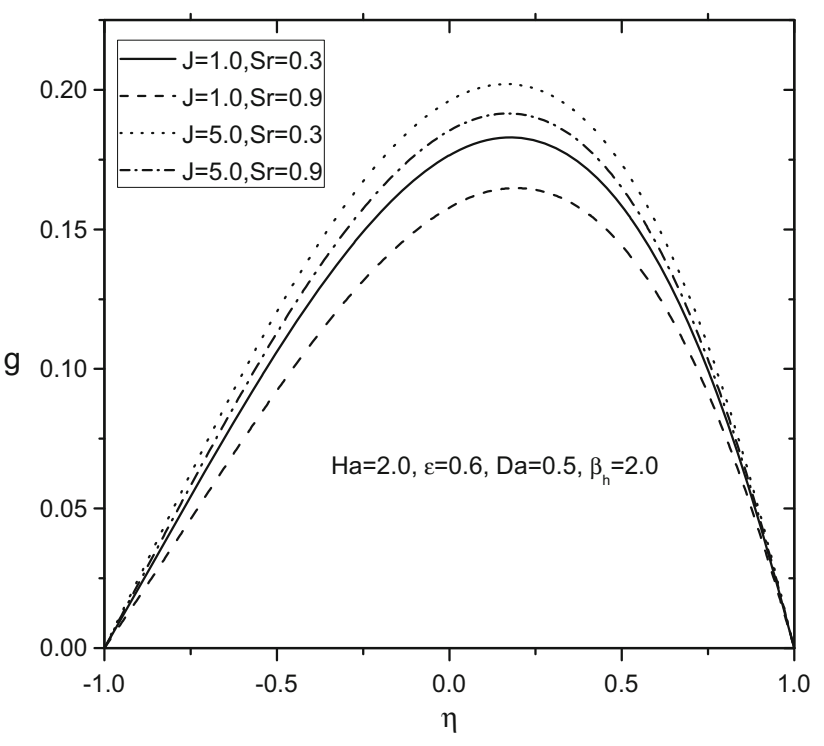

Fig. 7 Effect of $J$ and $\mathrm{Sr}$ on angular velocity profile

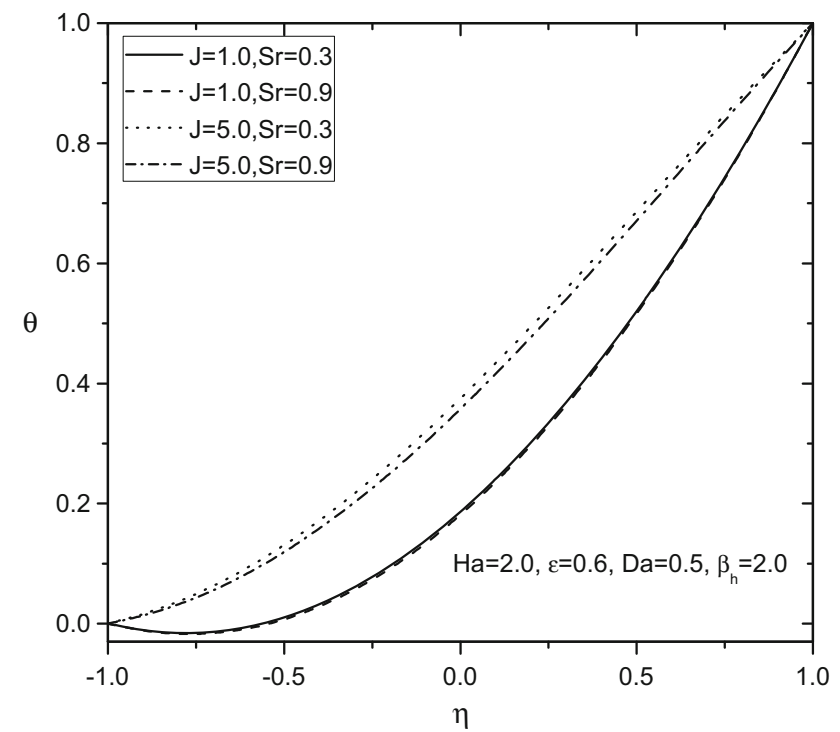

Fig. 8 Effect of $J$ and $\mathrm{Sr}$ on temperature profile

the influence of Hall number on concentration is shown in Fig. 5. It is observed from the figure that the dimensionless concentration increases with an increase in Hall parameter. This is due to the fact that decrease in temperature accelerates the concentration. In addition to the above, the effect of Darcy parameter on velocities, temperature and concentration profiles have been presented in Figs. 2, 3, 4 and 5 . it can be seen from these figures that the flow velocity $f(\eta)$ increases as Darcy number increases. It is observed from Fig. 3 that the induced velocity increases as Da increases. This is due to the fact that the lower permeability enhances the flow, which leads to increase in the velocities 


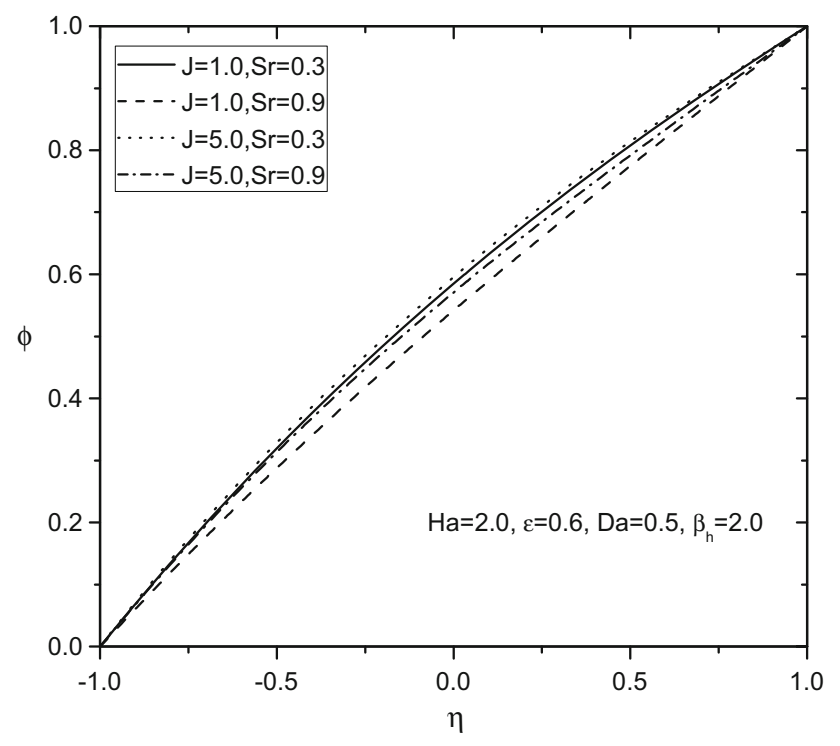

Fig. 9 Effect of $J$ and $\mathrm{Sr}$ on concentration profile

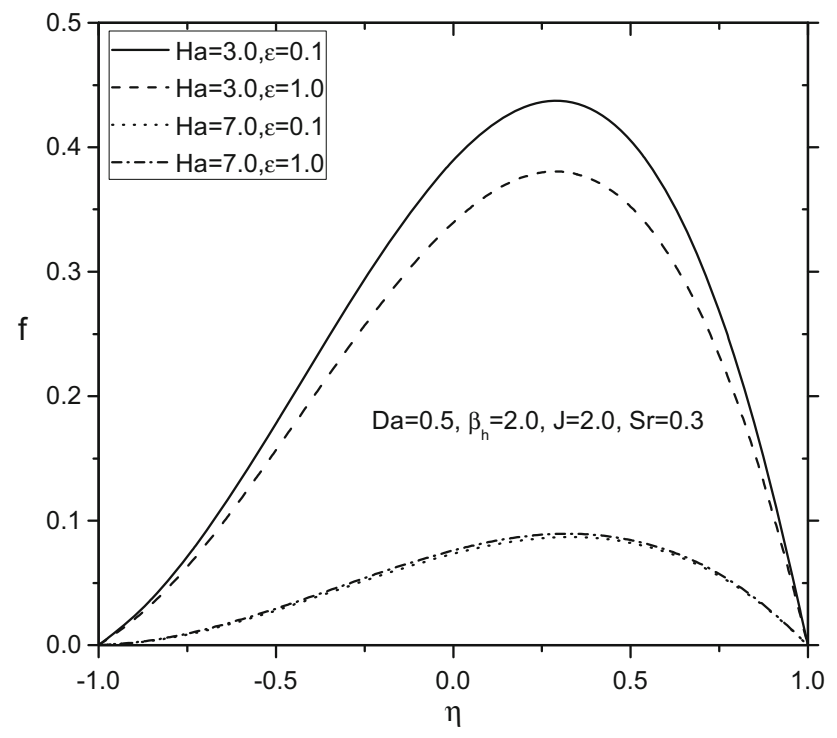

Fig. 10 Effect of Ha and $\varepsilon$ on velocity profile

of the flow. The influence of $\mathrm{Da}$ on temperature profile is shown in Fig. 4. It can be noted from this figure that as Da increases the dimensionless temperature increases. Finally the influence of Darcy number on concentration is shown in Fig. 5. It is observed from the figure that the dimensionless concentration increases with an increase in Darcy parameter. Increasing Da increases the porous medium permeability and simultaneously decreases the darcian impedance since progressively less solid fibers are present in the regime.

The influence of thermal diffusion parameter on velocities, temperature and concentration are shown in Figs. 6, 7, 8 and 9. It is clear from Figs. 6 and 7 that the higher values

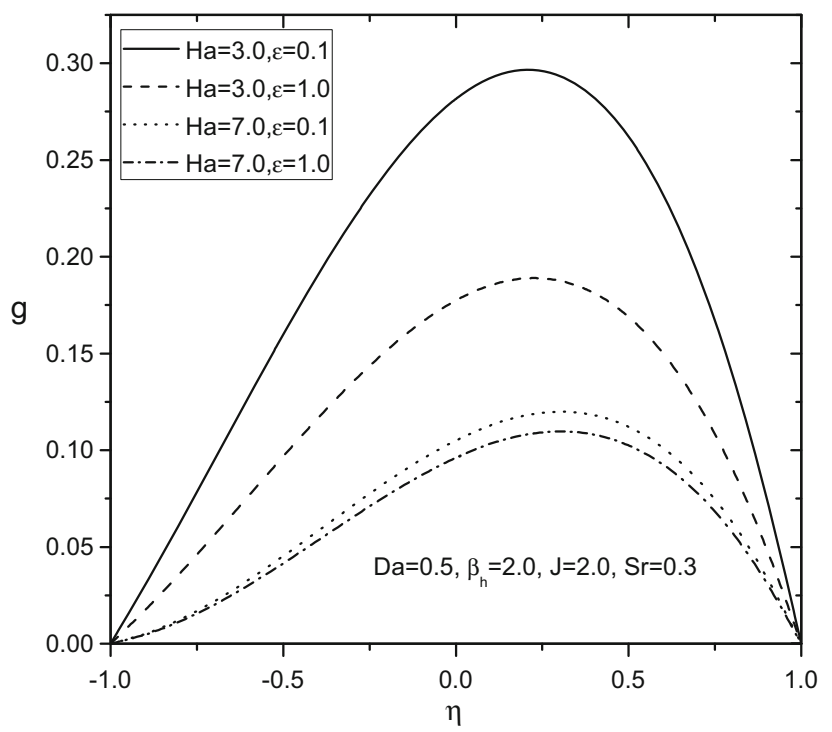

Fig. 11 Effect of Ha and $\varepsilon$ on angular velocity profile

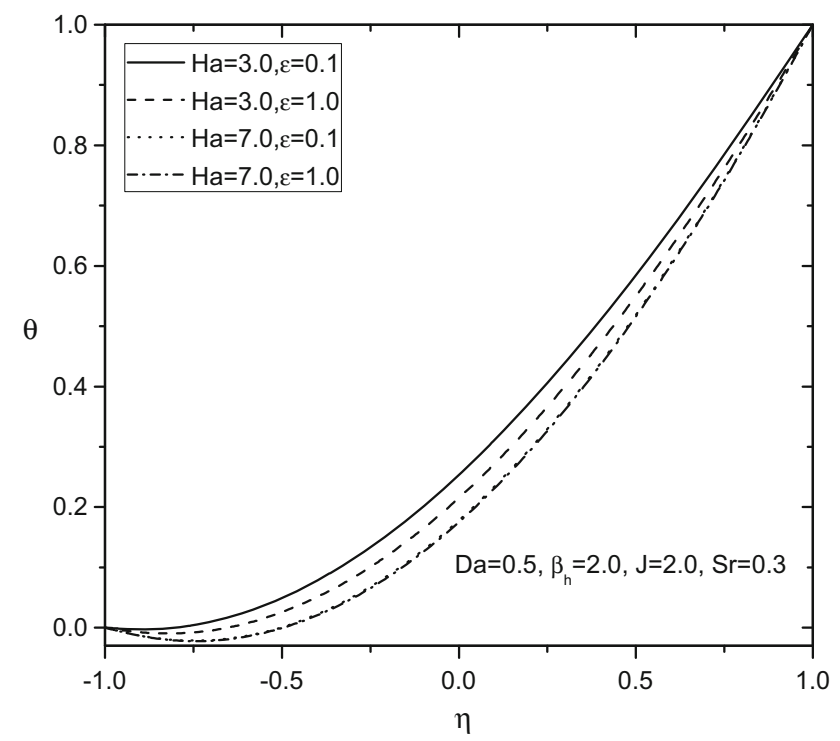

Fig. 12 Effect of Ha and $\varepsilon$ on temperature profile

of Soret number $\mathrm{Sr}$ decreases the velocities. Figure 8 presents the effect of $\mathrm{Sr}$ on dimensionless temperature. It is noticed from this figure that the temperature decreases with the higher values of Sr. Figure 9 explains the nature of the concentration profile for different values of Sr. It can be seen that the concentration of the fluid decreases as $\mathrm{Sr}$ increases. Figures 6, 7, 8 and 9 presents the influence of Joule heating parameter on velocities, temperature and concentration profiles. It can be seen from these figures as $J$ increases the velocity profiles decreases considerably. It is observed from Figs. 8 and 9 that the dimensionless temperature and concentration profiles decreases with an increase in $J$. 


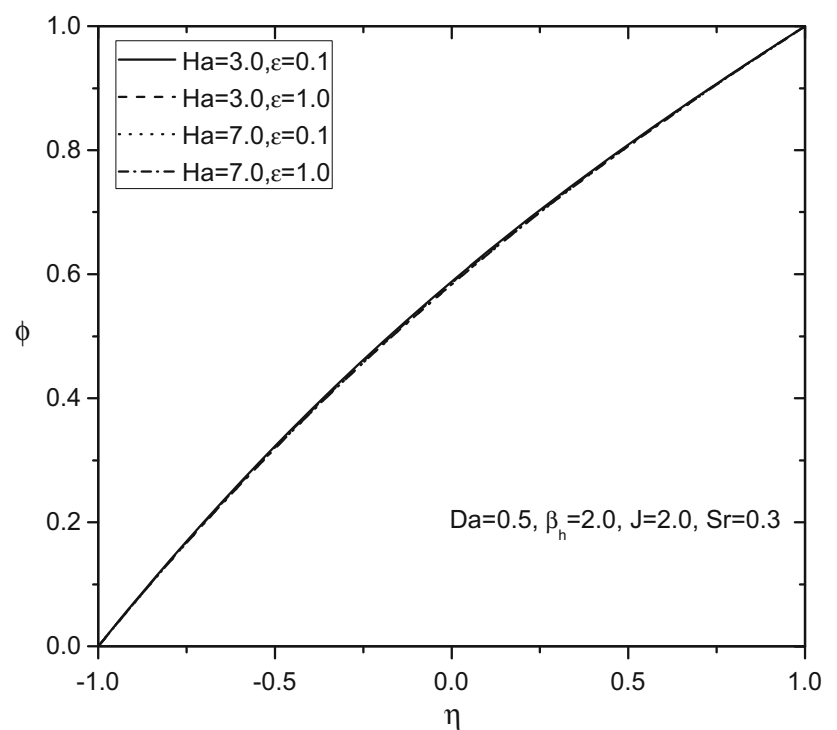

Fig. 13 Effect of Ha and $\varepsilon$ on concentration profile

Figure 10 prepared to study the influence of the Hartman number $\mathrm{Ha}$ and the porosity parameter $\epsilon$ on $f(\eta)$. It is seen from this figure that the flow velocity decreases when Ha increases. Since the flow is resisted by the Lorentz forces generated due the aaplied magnetic field. Figure 11 presents the influence of $\mathrm{Ha}$ on the cross flow velocity $g(\eta)$. By nature it has been noticed by many researchers that the cross flow velocity increases with the increase of Ha but it is noticed from the present study that the cross flow velocity decreases as the magnetic parameter increases. Porosity of the flow regime diminishes the concentration of the magnetic effect, which leads to the decreases the velocity in z-direction. The nature of dimensionless temperature has been presented in Fig. 12 with the various of Ha. It is observed from this figure that the temperature decreases with an increase in Ha. The effect of magnetic parameter on $\phi(\eta)$ is revealed in Fig. 13. It is seen from this figure that the concentration of the fluid decreases as Ha increases.

As discussed above, the Lorentz force creates resistance in the fluid which leads to the friction between the fluid layers. Hence the temperature and concentration of the fluid decreases with Magnetic parameter. In addition to the effect of magnetic parameter, porosity influence on velocities, temperature and concentrations are also been presented in Figs. 10 and 11. It is clear from the figures that

Table 1 Nature of skin friction coefficient, heat and mass transfer rates for various values of $\mathrm{Sr}, \mathrm{Ha}, \beta_{\mathrm{h}}, \epsilon, \mathrm{Da}$ and $J$ when $\mathrm{Re}=2.0, N=$ $5, \mathrm{Pr}=0.71, \mathrm{Gr}=1.0$ and $\mathrm{Sc}=0.22$

\begin{tabular}{|c|c|c|c|c|c|c|c|c|c|c|c|}
\hline $\mathrm{Ha}$ & $\epsilon$ & $\mathrm{Da}$ & $\beta_{\mathrm{h}}$ & $J$ & $\mathrm{Sr}$ & $C_{\mathrm{f}_{1}}$ & $C_{\mathrm{f}_{2}}$ & $\mathrm{Nu}_{1}$ & $\mathrm{Nu}_{2}$ & $\mathrm{Sh}_{1}$ & $\mathrm{Sh}_{2}$ \\
\hline 3 & 0.6 & 0.5 & 2 & 2 & 0.3 & 0.3261 & -2.5849 & 0.1050 & -0.9523 & -0.7096 & -0.3626 \\
\hline 5 & 0.6 & 0.5 & 2 & 2 & 0.3 & 0.0349 & -1.1900 & 0.1588 & -1.0228 & -0.7049 & -0.3662 \\
\hline 7 & 0.6 & 0.5 & 2 & 2 & 0.3 & 0.0046 & -0.5981 & 0.1846 & -1.0919 & -0.7018 & -0.3666 \\
\hline 9 & 0.6 & 0.5 & 2 & 2 & 0.3 & 0.0020 & -0.3457 & 0.1954 & -1.1325 & -0.7002 & -0.3663 \\
\hline 2 & 0.1 & 0.5 & 2 & 2 & 0.3 & 1.0086 & -4.2104 & 0.0311 & -0.8778 & -0.7152 & -0.3570 \\
\hline 2 & 0.4 & 0.5 & 2 & 2 & 0.3 & 0.8461 & -3.8124 & 0.0709 & -0.9468 & -0.7113 & -0.3590 \\
\hline 2 & 0.6 & 0.5 & 2 & 2 & 0.3 & 0.7551 & -3.5824 & 0.0901 & -0.9811 & -0.7094 & -0.3600 \\
\hline 2 & 0.8 & 0.5 & 2 & 2 & 0.3 & 0.6767 & -3.3771 & 0.1054 & -1.0089 & -0.7079 & -0.3607 \\
\hline 2 & 0.6 & 0.1 & 0 & 2 & 0.3 & 0.2663 & -2.0681 & 0.1730 & -1.1372 & -0.7011 & -0.3636 \\
\hline 2 & 0.6 & 0.3 & 2 & 2 & 0.3 & 0.6092 & -3.1933 & 0.1177 & -1.0317 & -0.7066 & -0.3613 \\
\hline 2 & 0.6 & 0.5 & 4 & 2 & 0.3 & 0.7551 & -3.5824 & 0.0901 & -0.9811 & -0.7094 & -0.3600 \\
\hline 2 & 0.6 & 1.0 & 6 & 2 & 0.3 & 0.8967 & -3.9377 & 0.0594 & -0.9265 & -0.7124 & -0.3585 \\
\hline 2 & 0.6 & 0.5 & 1 & 2 & 0.3 & 0.5362 & -2.9896 & 0.0779 & -0.8849 & -0.7130 & -0.3617 \\
\hline 2 & 0.6 & 0.5 & 3 & 2 & 0.3 & 0.9281 & -3.9401 & 0.1023 & -1.0385 & -0.7070 & -0.3595 \\
\hline 2 & 0.6 & 0.5 & 5 & 2 & 0.3 & 1.1013 & -4.2506 & 0.1155 & -1.0879 & -0.7048 & -0.3594 \\
\hline 2 & 0.6 & 0.5 & 7 & 2 & 0.3 & 1.1700 & -4.3647 & 0.1208 & -1.1059 & -0.7039 & -0.3594 \\
\hline 2 & 0.6 & 0.5 & 2 & 1 & 0.3 & 0.7146 & -3.5328 & 0.1519 & -1.0987 & -0.7032 & -0.3626 \\
\hline 2 & 0.6 & 0.5 & 2 & 3 & 0.3 & 0.7984 & -3.6353 & 0.0241 & -0.8563 & -0.7160 & -0.3571 \\
\hline 2 & 0.6 & 0.5 & 2 & 5 & 0.3 & 0.8956 & -3.7534 & -0.123 & -0.5806 & -0.7308 & -0.3505 \\
\hline 2 & 0.6 & 0.5 & 2 & 7 & 0.3 & 1.0113 & -3.8928 & -0.300 & -0.2597 & -0.7483 & -0.3422 \\
\hline 2 & 0.6 & 0.5 & 2 & 2 & 0.3 & 0.7551 & -3.5824 & 0.0901 & -0.9811 & -0.7094 & -0.3600 \\
\hline 2 & 0.6 & 0.5 & 2 & 2 & 0.5 & 0.7009 & -3.5222 & 0.0947 & -0.9888 & -0.6853 & -0.3884 \\
\hline 2 & 0.6 & 0.5 & 2 & 2 & 0.7 & 0.6454 & -3.4612 & 0.0992 & -0.9965 & -0.6606 & -0.4171 \\
\hline 2 & 0.6 & 0.5 & 2 & 2 & 0.9 & 0.5888 & -3.3992 & 0.1036 & -1.0040 & -0.6353 & -0.4462 \\
\hline
\end{tabular}


the velocity profiles $f(\eta), g(\eta)$, the temperature $(\theta(\eta))$ and the concentration of the fluid flow decreases with an increase in $\epsilon$. This can be attributed to the fact that increasing $\epsilon$ decreases the velocities and, in turn, decreases the viscous dissipation which decreases the temperature and concentration.

Variation of Joule heating parameter $(J)$, thermal diffusion parameter (Sr), magnetic parameter $\mathrm{Ha}$, Hall number $\left(\beta_{\mathrm{h}}\right)$, porosity parameter $(\epsilon)$ together with the Darcy parameter $(\mathrm{Da})$ is presented in Table 1 with fixed values of other parameters. It can be seen from this table that the skin friction coefficient decreases at the initial plate and increases at the terminal plate where as the reverse trend is noticed on heat and mass transfer rate with an increase in $\mathrm{Ha}$ and $\epsilon$. As magnetic parameter increases, the resistive force slow downs the friction factor at $\eta=-1$. It is observed that as porosity of the medium increases, heat transfer rate and mass transfer rates increases at $\eta=-1$ and decreases at the other plate but the skin friction coefficient presents the reverse trend. It is clear from the table that the friction, heat and mass transfer rates are increases at the initial plates and decreases at the terminal plate with the increase of Hall parameter, Joule heating parameter and Da. Finally, the influence of $\mathrm{Sr}$ on friction factor, heat and mass transfer rates are presented in this table. The performance of these parameters are self-evident from the Table 1 and hence are not discussed for brevity. These results are clearly shows that the emerging parameters have remarkable impact on all the profiles.

\section{Conclusions}

This present study investigates the steady manetohydrodynamic flow of newtonian fluid in a vertical channel saturated porous medium in presence of Hall, Joule heating and the Soret effects. Adomian decomposition method is used to solve the final dimensionless governing equations. The significant findings are summarized as:

- Fluid flow velocity and the concentration profiles amplifies where as the flow in $z$-direction and the the temperature profile decreases with an increase in Ha.

- As Da increases, the velocity profiles, temperature profile and concentration profile are increased.

- It is noticed that the presence of Soret and Joule heating parameters in the fluid decreases the velocities, temperature and the concentration of the fluid.

- The velocities, temperature and concentration profiles are decreases with the increase in the magnetic and porosity parameters.
Open Access This article is distributed under the terms of the Creative Commons Attribution 4.0 International License (http://crea tivecommons.org/licenses/by/4.0/), which permits unrestricted use, distribution, and reproduction in any medium, provided you give appropriate credit to the original author(s) and the source, provide a link to the Creative Commons license, and indicate if changes were made.

\section{References}

1. Ingham, D.B., Pop, I., Cheng, P.: Combined free and forced convection porous medium between two vertical walls with viscous dissipation. Transp. Porous Media 5, 381-398 (1990)

2. Paul, T., Jha, B.K., Singh, A.K.: Free-convection between vertical walls partially filled with porous medium. Heat Mass Transf. 33, 515-519 (1998)

3. Umavathi, J.C.: Free convection of composite porous medium in a vertical channel. Heat Transf. Asian Res. 40(4), 308-329 (2011)

4. Mishra, A.K., Djam, X.Y., Manjak, N.H.: Effect of radiation on free convection flow due to heat and mass transfer through porous medium bounded by two vertical walls. Int. J. Adv. Technol. Eng. Res. 3, 120-125 (2013)

5. Afify, A.A.: Similarity solution in MHD: effects of thermal diffusion and diffusion thermo on free convective heat and mass transfer over a stretching surface considering suction or injection. Commun. Nonlinear Sci. Numer. Simul. 14, 2202-2214 (2009)

6. Srinivasacharya, D., Kaladhar, K.: Soret and Dufour effects on free convection flow of a couple stress fluid in a vertical channel with chemical reaction. Chem. Ind. Chem. Eng. Q. 19(1), 45-55 (2013)

7. Srinivasacharya, D., Pranitha, J., Ramreddy, C.H., Postelnicu, A.: Soret and Dufour effects on non-Darcy free convection in a power-law fluid in the presence of a magnetic field and stratification. Heat Transf. Asian Res. 43, 592-606 (2014)

8. Tani, I.: Steady flow of conducting fluids in channels under transverse magnetic fields with consideration of Hall effects. J. Aerosp. Sci. 29, 297-305 (1962)

9. Srinivasacharya, D., Shiferaw, M.: Hall and Ion-slip effects on the flow of micropolar fluid between parallel plates. Int. J. Appl. Mech. Eng. 13(1), 251-262 (2008)

10. Srinivasacharya, D., Shiferaw, M.: MHD flow of a micropolar fluid in a rectangular duct with Hall and Ion-slip effects. J. Braz. Soc. Mech. Sci. Eng. 30(4), 313-318 (2008)

11. Srinivasacharya, D., Shiferaw, M.: MHD flow of a micropolar fluid in a circular pipe with Hall effects. ANZIAM J. 51, 277-285 (2009)

12. Manglesh, A., Gorla, M.G.: MHD free convection flow through porous medium in the presence of Hall current, radiation and thermal difusion. Indian J. Pure Appl. Math. 44(6), 743-756 (2013)

13. Garg, B.P., Singh, K.D., Bansal, A.K.: Hall current effect on viscoelastic (Walters liquid model-B) MHD oscillatory convective channel flow through a porous medium with heat radiation. Kragujev. J. Sci. 36, 19-32 (2014)

14. Chen, C.H.: Combined effects of Joule heating and viscous dissipation on magnetohydrodynamic flow past a permeable, stretching surface with free convection and radiative heat transfer. J. Heat Transf. 132, 064503-1-064503-5 (2010)

15. Hossain, M.A., Gorla, R.S.R.: Joule heating effect on magnetohydrodynamic mixed convection boundary layer flow with variable electrical conductivity. Int. J. Numer. Methods Heat Fluid Flow 23(2), 275-288 (2013) 
16. Nandkeolyar, R., Motsa, S.S., Sibanda, P.: Viscous and Joule heating in the stagnation point nanofluid flow through a stretching sheet with homogenous- heterogeneous reactions and nonlinear convection. J. Nanotechnol. Eng. Med. 4, 0410011-1-0410011-9 (2013) 